\title{
Epidemiology of Impaired Renal Function in the Bamenda Health District, North West Region of Cameroon
}

\author{
Tetuh KM ${ }^{1}$, Thomas $\mathrm{VA}^{2}$, Fomboh $\mathbf{R}^{1,3^{*}}$, Yongsi $\mathrm{BN}^{4}$, Mankolo $\mathrm{BO}^{5}$ and Alberic $\mathbf{S}^{1}$ \\ ${ }^{1}$ Value Health Africa, Bamenda, Cameroon \\ ${ }^{2}$ Division of Health Operations Research, Ministry of Public Health, Cameroon \\ ${ }^{3}$ Higher Institute of Medicine and Biomedical Sciences, Cameroon Christian University, Bali, Cameroon \\ ${ }^{4}$ Faculty of Science, University of Yaoundel, Cameroon \\ ${ }^{5}$ Coordinator of the project MTN FAIRMED, Cameroon
}

*Corresponding Author: Fomboh Richard, Higher Institute of Medicine and Biomedical Sciences, Cameroon Christian University (CCU), Cameroon, Tel: (+237) 674440289; E-mail: drfombohrich@gmail.com

Received date: Aug 18, 2017; Accepted date: Sep 5, 2017; Published date: Sep 11, 2017

Copyright: ( 2017 Tetuh KM, et al. This is an open-access article distributed under the terms of the Creative Commons Attribution License, which permits unrestricted use, distribution, and reproduction in any medium, provided the original author and source are credited.

\begin{abstract}
Impaired renal function (IRF) is a worldwide problem with adverse outcome of kidney failure, cardiovascular disease (CVD) and death. Consequently research on assessing the burden of this disease in a developing Country like Cameroon where sustainability of end-stage management cannot be guaranteed, is crucial for the adoption of better prevention strategies. The objective of this study was to describe the epidemiology of Impaired Renal Function, among adults in Bamenda health district by the end of 2015. It was a cross sectional study that lasted from March 2015 to December 2015 at the Bamenda Health District. Blood samples were collected from 512 participants through a stratified convenience sampling technique. IRF was defined as Egfr $\leq 60 \mathrm{ml} / \mathrm{min} / 1.73 \mathrm{~m}^{2}$. Data was analysed using Epi Info version 3.5.1 and Microsoft Excel 2010. Out of the 512 participants, there were 305 (59.6\%) females and $207(40 \%)$ males of which $294(57.4 \%)$ were from the rural communities and $218(42.6 \%)$ from the urban communities. The mean age of the participants was 52 years $(S D=15.43)$. It was observed that the prevalence of new cases of the various diseases was very high; hypertension was $44.1 \%$, IRF was $7.40 \%$ and Diabetes was $6.4 \%$. This result could be correlated with other studies in Africa. There was also a prevalence of $0.8 \%$ of participants who were already at stage 5 but who weren't aware of their status. Prevalence of reduced eGFR was high in the entire population and predominatly dominated by the rural population while awareness was low. Factors associated to IRF were obesity $(\mathrm{AOR}=2.39 ; 95 \% \mathrm{Cl} 1.13-5.06)$; pre-diabetes $(\mathrm{AOR}=2.53 ; 95 \% \mathrm{Cl} 1.03-6.21)$ and age $(A O R=1.04 ; 95 \% \mathrm{Cl} 1.01-1.07)$. IRF is an increasing burden in Cameroon. More research needs to be done so as to identify some of the risk factors of every community for better interventions.
\end{abstract}

Keywords: Impaired renal function; Epidemiology; Prevalence; Community; Bamenda

\section{Introduction}

Impaired Renal Function (kidney disease or kidney malfunctions), is a worldwide problem with adverse outcome of kidney failure, cardiovascular disease (CVD) and death [1]. According to the 2010 Global Burden of Disease study, Chronic kidney Disease (CKD) was ranked $27^{\text {th }}$ in the list of causes of total number of global deaths in 1990 (age-standardized annual death rate of 15.7 per 100000 ), but rose to $18^{\text {th }}$ in 2010 (annual death rate $16 \cdot 3$ per 100000 ) with a prevalence estimate of $10 \%$ worldwide while the incidence and prevalence of acute kidney injury globally are still unknown, due to the use of different definitions [2] and the overlap it has with CKD. The incidence of endstage renal disease worldwide is consistently rising, currently at the rate of $6 \%$ per year [3] which is much higher than the population growth in the world, estimated at $1.2 \%$ yearly.

Even though statistics for most of the developing countries are unknown [4], following a research on CKD in Senegal, Tanzania and Congo [5], results indicated that the prevalence of CKD in these countries were respectively $6.1 \%, 7.0 \%$ and $13.2 \%$ [6,7] which is still very high considering the GDP of these countries. The situation in
Sub-Saharan Africa (SSA) is even more dismal and worsened by the almost complete lack of data making it difficult for policy makers to see the gravity of the disease [3]. This disease could be a ticking time bomb for the entire region, bearing in mind that large proportions of the people in this region live below the poverty level of $\$ 1$ per day [8].

In Sub-Saharan Africa alone, the number of people with diabetes is projected to increase from 7 million in 2000 to 18 million in 2030, a regional increase of $161 \%$ [9]. Additionally, complications of diabetes mellitus are more prevalent among patients with diabetes in Africa as compared to the developed world due to late presentation, limited screening and diagnostic resources, poor glycaemic control, and inadequate treatment of complications at an early stage [10]. Screening for CKD is not routinely performed in many diabetic clinics in SubSaharan Africa due to limited diagnostic resources. In particular, micro albumin testing is available in very few centres in developing countries, the delayed diagnosis of patients at end-stage renal disease (ESRD) has a limited interest because many patients at the end will not have access to renal replacement therapy $[11,12]$ and the cost of health services for patients with CKD is 1.8 times higher compared to patients without $\mathrm{CKD}$, and the average cost for a dialysis patient is 10.3 times more than that of a non-CKD patient [13].

Added to this, $80 \%$ of low human development countries (countries with high population growth, low income, low literacy, and low life 
expectancy) are in Africa [3], the resultant effect of this is the combined increase in the prevalence of communicable and noncommunicable diseases, which are often undetected until late and, worse still, poorly managed even when detected. Kidney disease is an important cause of death and loss of disability-adjusted life-years but awareness is low among patients and health-care providers [1]. Although decreased GFR can be caused by primary kidney disease (predominantly glomerular diseases, tubulo-interstitial diseases, obstruction, and polycystic kidney disease), in the vast majority of patients with decreased GFR, the kidney damage is associated with other medical conditions such as diabetes and hypertension [14].

In a study to assess the prevalence and correlates of CKD in an urban adults Cameroonian population by [14] using the commonest estimators of kidney function, there was a high frequency of CKD and related risk factors in this population, with over one in ten participants having CKD. This high prevalence of CKD appeared to be driven mostly by advanced age, hypertension, diabetes mellitus and adiposity.

In Cameroon, developing prevention and management programs are still difficult due to the scarcity of epidemiologic data at population level (community based research) and scarcity of resources making it difficult for policy makers to see the gravity of the problem [15]. Just like in most other SSA countries, nephrology services in Cameroon are not widely available and are mostly understaffed. In 2012 for instance, the country had a total of five nephrologists who were all practicing in the two main cities of the country (Douala and Yaounde). In this same year the country had eight hemodialysis centers providing care to about 500 patients with ESRD [16]. To that effect, there have been an increasing number of people with various stages of IRF reporting to or referred to public institutions with dialysis facilities which have provided opportunities for the characterization of some specific segments of the populations with $\mathrm{CKD}$, in the absence of large community based-studies [17]. Hence most estimates and statistics on CKD studies in Cameroon have been gotten from hospital based research, indicating the urgent need for a community based approach which provides a clearer image on the state of the disease in the entire country. We therefore seek to determine the prevalence, associated risk factors (BHD) and the level of awareness of (IRF) in the Bamenda Health District.

\section{Methodology}

A Community based cross-sectional descriptive and analytic study was carried out from March- December 2015 in the Bamenda health district. Adults aged 20 years and above of both sexes who gave their consent were recruited into the study. Persons less than 20 years of age (for convenient) and pregnant women were excluded from the study. A total of 512 participants were recruited into the study. Data was collected using standardize questionnaires adapted for the purpose of this study and were administered to participants (we are talking of adults here $>20$ years). The questionnaire was structured in six sections viz; socio-demographic data, Health history, Habits, Awareness, measurement of anthropometric parameters (weight, height or length and waist circumference), Laboratory results of Diabetes, hypertension and Kidney function. The screening was conducted by trained medical personnel. The physical examination included BP and anthropometric measurements. BP (systolic and diastolic) was measured using a standardized protocol with the participant in a seated position, and after at least $30 \mathrm{~min}$ of rest. BP measurements were performed on the right arm using sphygmomanometers). Special attention was given to the use of appropriate cuff sizes $(13 \times 23 \mathrm{~cm}$ or $16 \times 30 \mathrm{~cm})$. Weight (to the nearest $0.5 \mathrm{~kg}$ ) was measured with the use of an automated scale. Participants were permitted to keep on light clothing. Height (in metres to the nearest $0.5 \mathrm{~cm}$ ) was measured using a wooden platform and a height rule. Body mass index $\left(\mathrm{BMI}\right.$ in $\left.\mathrm{kg} / \mathrm{m}^{2}\right)$ was calculated as weight $\{(\mathrm{kg}) /($ height $(\mathrm{m}) \times$ height $(\mathrm{m})\}$.

The sociodemographic and clinical Data was entered and analysed using Epi-Info version 3.5.1 and Excel 2010. Descriptive and inferential statistical analysis was used. Bivariate and multivariate logistic regression was used to determine the association between IRF and other variables.

IRF was the dependent variable and other variables such as diabetes, hypertension, age, alcohol, obesity etc were considered as the independent variable. Unadjusted and adjusted odd ratio and their corresponding $95 \%$ confidence intervals were used to examine the strength of association. P-value $<0.05$ was considered statistically significant. For the main analyses, prevalence and determinants of IRF are based on MDRD derived from eGFR. Ethical clearance was obtained from the National Ethical Review Board. Research authorization was obtained from the Bamenda Health District. Informed verbal and written consent were obtained from the participants after briefing them on the objectives of the study.

\section{Results}

Out of the 512 participants 305 (59.6\%) were females and 207 (40\%) were males and of which $294(57.4 \%)$ were from the rural communities and $218(42.6 \%)$ from the urban communities. The mean age of the participants was 52 years $(\mathrm{SD}=15.43)$ with the youngest participant being 20 years and the oldest 90 years of age. The most represented age group was the 52-59 years age group, and the least represented age group was the 84-91 years age group as in Table 1 . Approximately 328 (64.3\%) of these participants were within the active working population of the society (20-60 years) as in Table 1 . Majority of the participants had primary education $(36.30 \%)$.

We observed the prevalence of various diseases amongst the participants with hypertension being the highest (54.5\%) as in figure 1 and CKD (is it IRF or CKD) the lowest with $7.40 \%$ as in Table 3. Obesity, Hypertension and age were associated to chronic kidney disease under binary logistic regression, after multivariate logistic regression, obesity and age remained associated to chronic kidney disease.

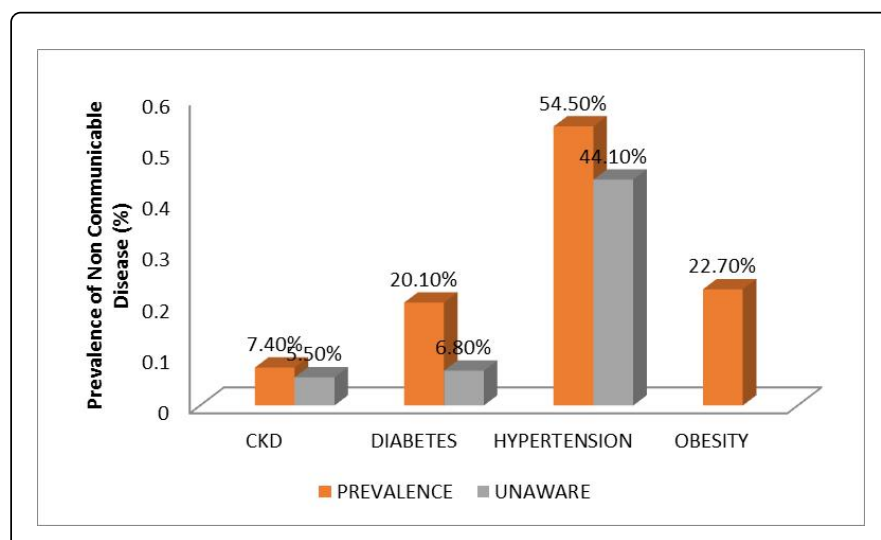

Figure 1: Prevalence of various diseases among study population. 
Citation: Tetuh KM, Thomas VA, Fomboh R, Yongsi BN, Mankolo BO, et al. (2017) Epidemiology of Impaired Renal Function in the Bamenda Health District, North West Region of Cameroon. J Kidney 3: 148. doi:10.4172/2472-1220.1000148

Page 3 of 5

Socio-demographic characteristics

\begin{tabular}{|c|c|c|c|c|}
\hline Characteristics & Male $\mathbf{N}=207(\%)$ & $\begin{array}{l}\text { Female } \\
\mathrm{N}=305(\%)\end{array}$ & $\begin{array}{l}\text { Total } \\
\mathrm{N}=512(\%)\end{array}$ & P-value \\
\hline Mean Age & $51.06 \pm 15.76$ & $\begin{array}{l}53.96 \\
15.12\end{array}$ & 512 & 0.03 \\
\hline $20-27$ & 21 & 19 & 40 & 0.1 \\
\hline $28-35$ & 15 & 18 & 33 & 0.93 \\
\hline $36-43$ & 26 & 18 & 60 & 0.87 \\
\hline $44-51$ & 35 & 47 & 82 & 0.33 \\
\hline $52-59$ & 45 & 64 & 109 & 0.53 \\
\hline $60-67$ & 31 & 57 & 88 & 0.18 \\
\hline $68-75$ & 24 & 46 & 70 & 0.04 \\
\hline $76-83$ & 7 & 16 & 23 & 0.02 \\
\hline $84-91$ & 3 & 4 & 7 & 0.27 \\
\hline \multicolumn{5}{|c|}{ Educational Level } \\
\hline None & $21(10.1)$ & $134(43.9)$ & 155 & 0.12 \\
\hline Primary & $81(39.1)$ & $105(34.4)$ & 186 & 0.45 \\
\hline Secondary & $34(16.4)$ & $18(5.9)$ & 52 & 0.12 \\
\hline High school & $22(10.6)$ & $13(4.3)$ & 35 & 0.81 \\
\hline University & $49(23.7)$ & $35(11.5)$ & 84 & 0.89 \\
\hline
\end{tabular}

\begin{tabular}{|c|c|c|c|c|}
\hline \multicolumn{5}{|l|}{ Monthly Income } \\
\hline No Salary & $96(46.4)$ & $232(76.1)$ & 328 & 0.23 \\
\hline$<50,000$ & $54(26.1)$ & $46(15.1)$ & 100 & 0.67 \\
\hline $51000-100,000$ & $31(15)$ & $15(4.9)$ & 46 & 0.74 \\
\hline $110-250,000$ & $15(7.2)$ & $8(2.6)$ & 23 & 0.54 \\
\hline$>251000$ & $11(5.3)$ & $4(1.3)$ & 15 & 0.59 \\
\hline \multicolumn{5}{|l|}{ Residence } \\
\hline Rural & $78(37.7)$ & $\begin{array}{l}216 \\
(70.8 \%)\end{array}$ & 294 & 0.27 \\
\hline Urban & $129(62.3)$ & $89(29.2 \%$ & 218 & 0.23 \\
\hline
\end{tabular}

Table 1: Socio-demographic characteristics.

\section{Clinical characteristics}

According to the result, there was no significant different in the number of participants with history of diabetes and history of IRF in terms of gender; meanwhile for those with history of hypertension, there were more females (37.6\%) than males $(24.5 \%)(\mathrm{P}-0.002)$. It is likely that women are more at risk of hypertension than their male counterparts. Correlating the usage of Herbal medicine with the appearance of IRF, it shows that their correlation was not statistically significant (Table 2). Therefore, herbal medicine does not play any role in the appearance of IRF.

\begin{tabular}{|l|l|l|l|l|}
\hline Characteristic & Male N=207(\%) & Female N=305(\%) & Total N (\%) & P-Value \\
\hline History of Diabetes & $28(13.6)$ & $39(12.9)$ & $67(13.2)$ & 0.45 \\
\hline History of Hypertension & $49(24.5)$ & $92(37.6)$ & $141(31.7)$ & 0.002 \\
\hline History of IRF & $15(7.5)$ & $15(6.1)$ & $30(6.8)$ & 0.34 \\
\hline Use of Herbal Medicine & $125(62.8)$ & $190(64.0)$ & $315(63.5)$ & 0.43 \\
\hline Smoking & $19(9.2)$ & $24(7.9)$ & $43(8.4)$ & 0.36 \\
\hline Alcohol & $162(78.3)$ & $179(59.3)$ & $341(67.0)$ & $<0.0001$ \\
\hline
\end{tabular}

Table 2: Clinical characteristics of study population.

\section{Prevalence and Severity of IRF}

\begin{tabular}{|l|l|l|l|l|}
\hline Prevalence of IRF & Male N(\%) & Female N(\%) & Total N(\%) & P-value \\
\hline IFR<60 & $12(5.8)$ & $26(8.5)$ & $38(7.4)$ & 0.16 \\
\hline STAGE 3A & $6(2.9)$ & $16(5.2)$ & $22(4.3)$ & 0.25 \\
\hline STAGE 3B & $5(2.4)$ & $5(1.6)$ & $10(2)$ & 0.34 \\
\hline STAGE 4 & $0(0)$ & $2(0.7)$ & $2(0.4)$ & 0.45 \\
\hline STAGE 5 & $1(0.5)$ & $5(1)$ & $4(0.8)$ & 0.67 \\
\hline
\end{tabular}

Table 3: Prevalence and classification of IRF. 
From Table 3 the prevalence of IRF was $7.4 \%$ with no significant difference in terms of gender ( $p$-value $=0.16$ ). The prevalence for new cases of Diabetes as associated disease to IRF was $6.4 \%$, with no significant different between males and females. Hypertension was
$44.1 \%$ and obesity, 36.5 (pre-obesity) and 15\% (Obesity class 1). There was a significant different between males and females with pre-obesity (Table 4).

\begin{tabular}{|l|l|l|l|l|}
\hline Disease & Male N(\%) & Female N(\%) & Total N(\%) & P-Value \\
\hline Diabetes & $50(17.4)$ & $53(24.2)$ & $105(20.1)$ & 0.03 \\
\hline New Cases & $20(9.7)$ & $13(4.3)$ & $33(6.4)$ & 0.01 \\
\hline Mean FBS & $102.57 \pm 51.39$ & $94.93 \pm 66.44$ & $97.69 \pm 60.69$ & $79(15.4)$ \\
\hline Pre-Diabetic & $40(19.3)$ & $39(12.8)$ & $279(54.5)$ & 0.16 \\
\hline Hypertension & $114(55.1)$ & $165(54.1)$ & $226(44.1)$ & 0.03 \\
\hline New Cases & $97(42.1)$ & $126(46)$. & $259(50.6)$ & 0.44 \\
\hline Pre Hypertension & $106(51.2)$ & $153(50.2)$ & $138(27)$ & 0.17 \\
\hline Stage 1 & $65(31.4)$ & $73(23.9)$ & $100(19.5)$ & 0.44 \\
\hline Stage 2 & $38(18.4)$ & $62(20.3)$ & $116(22.7)$ & 0.03 \\
\hline Obesity & $30(14.5)$ & $86(28.2)$ & $187(36.5)$ & 0.33 \\
\hline Pre-obesity & $93(44.9)$ & $94(30.8)$ & $77(15.0)$ & 0.0001 \\
\hline Obesity class 1 & $25(12.1)$ & $52(17.0)$ & $27(5.3)$ & 0.0008 \\
\hline Obesity class 2 & $2(1.0)$ & $25(8.2)$ & $12(2.3)$ & 0.07 \\
\hline Obesity class 3 & $3(1.4)$ & $9(3.0)$ & 0.0001 \\
\hline
\end{tabular}

Table 4: Distribution and classification of diseases associated to IRF in the study population.

\section{Discussion}

This study revealed a prevalence of decreased eGFR of $7.4 \%$ which was closer to the result obtained in Senegal (with a prevalence of 6.4\%). However we had a lower prevalence as opposed to previous research conducted in Dschang health District with a decreased eGFR prevalence of $13.2 \%$, which could however be explained by the difference in the methodology used. Our study also revealed a very high prevalence of Hypertension which corresponded to the prevalence gotten from a previous research in Cameroon with similar survey as ours [18]. The prevalence of newly diagnosed Diabetic and Obese cases were also very high and this prevalence corresponded to previous studies conducted in Cameroon [19].

Our study also revealed a high level of unawareness amongst people with hypertension and CKD with $44.1 \%$ of hypertensive patient unaware of their status while just $1.9 \%$ of the total CKD patients were aware of their condition which could be confirmed by the low level of CKD awareness in Cameroon and Africa [13]. However, although the diabetes prevalence was high, only $6 \%$ of the diabetes patients were not aware of their status indicating the success of most sensitization and prevention programs against diabetes but then more work still needs to be done in order to identify this $6 \%$ from the communities. Conversely, the awareness of CKD amongst diabetic patients were very low which could be attributed to the fact that, most community health running diabetes clinics don't have the capacity to measure GFR and hence refer these patients to the district level. Unfortunately, most of these patients do not comply with this due to accessibility financial constrains consequently; most of these patients are rushed to these referral hospitals only at end stage. This high prevalence can further be confirmed by the WHO 2035 projection which forecast a high burden of chronic diseases on the developing nations [12].

This high prevalence in Cameroon and Africa is due largely to unawareness, lack of personals and policy to prevent and manage these diseases. The situation is even more dismal and worsened by the almost complete lack of data making it difficult for policy makers to see the gravity of the disease [3]. Bearing in mind that large proportions of the peoples in this region live below the poverty level of $\$ 1$ per day [9], it could be realized that chronic diseases is not only a problem of the developed nations but it is presently serious problem globally and it now becoming a threat to developing countries especially as there is a steep transition in lifestyle as demonstrated by our theory (epidemiological transition theory). It becomes more appalling as Cameroon is a victim of both a high prevalence in Communicable diseases and non-communicable diseases-with much of its health resources invested in the fight against diseases like HIV/AIDS, Tuberculosis, Poliomyelitis, etc. and on the other hand there is a rising need for the investment on managing non-communicable diseases like hypertension, ESRD and diabetes. It is for this reason that there is an urgent need for better strategies to be put in place for the prevention of these non-communicable diseases since managing complications arising from these diseases have not been shown to be cost effective and sustainable [19]. In the case of Bamenda, it has been revealed that patients now undergo dialysis just twice weekly due to the lack of resources as opposed to thrice weekly, stipulated by the ideal. This is a clear indication that, with the rise in the prevalence of $\mathrm{CKD}$, the future 
Citation: Tetuh KM, Thomas VA, Fomboh R, Yongsi BN, Mankolo BO, et al. (2017) Epidemiology of Impaired Renal Function in the Bamenda Health District, North West Region of Cameroon. J Kidney 3: 148. doi:10.4172/2472-1220.1000148

Page 5 of 5

might not be a very good one for Cameroon if measures aren't put in place to arrest this situation.

\section{Conclusion}

Prevalence of reduced eGFR was high in the entire population and predominantly dominated by the rural population while awareness was low. Factors associated to IRF were obesity (AOR=2.39; $95 \%$ ci 1.13-5.06); pre-diabetes $(\mathrm{AOR}=2.53 ; 95 \% \mathrm{ci} 1.03-6.21)$ and age $(\mathrm{AOR}=1.04 ; 95 \% \mathrm{ci} 1.01-1.07)$. IRF is an increasing burden in Cameroon. More research needs to be done so as to identify some of the risk factors of every community for better interventions.

\section{Recommendation}

The Government should increase the capacity of the health centers to manage these non-communicable diseases by providing up-to-date training sessions to the health care providers on how these diseases could be managed. Considering the national burden of CKD, Hypertension, Diabetes, and Obesity and the impact of globalization on contemporary lifestyles and health, the need for international collaboration is great. Practical networks sharing common guidelines, but adapted to local cultures in a flexible way, have proved to be very useful. The government should also put up a health program for chronic kidney disease as the case is with HIV/AIDS while also facilitate the training of more Nephrologists for the management of CKD.

\section{Reference}

1. Courser WG, Remuzzi G, Mendis S, Tonelli M (2011) The Contribution of Chronic Kidney Disease to the Global Burden of Major noncommunicable Diseases. Kidney Int 80: 1258-1270.

2. Lancet (2015) GBD 2013 Mortality and Causes of Death Collaborators. Global, regional and national age-sex specific all-cause and cause-specific mortality for 240 causes of death, 1990-2013: A systematic analysis for the Global Burden of Disease Study 2013. Lancet 385: 117-171.

3. Bamgboye EL (2006) End-Stage Renal Disease in Sub-Saharan Africa. Ethn Dis 16.

4. Alebiosu CO, Ayodele OE (2005) The global burden of chronic kidney disease and the way forward. Ethn Dis 15: 418-423.

5. Stanifer WJ, Jing B (2014) The epidemiology of chronic kidney disease in sub-Saharan Africa: A systematic review and meta-analysis. The Lancet Global Health Blog.
6. Seck SM, Doupa D, Gueye L, Dia CA (2014) Prevalence of Chronic Kidney Disease and Associated Factors in Senegalese Populations: A Community-Based Study in Saint-Louis. Nephrourol Mon 6: 19085.

7. Stanifer JW, Maro V, Egger J, Karia F, Thielman N, et al. (2015) The Epidemiology of Chronic Kidney Disease in Northern Tanzania: A Population-Based Survey. PLoS One

8. Hunsicker L (2004) The consequences and costs of chronic kidney disease before ESRD. J Am Soc Nephrol 15: 1363-1364.

9. Gill GV, Mbanya JC (2009) A sub-Saharan African perspective of diabetes. Diabetologia 52: 8-16.

10. Barsoum R (2006) Chronic kidney disease in the developing world. N Engl JMed 354: 997-999.

11. WHO (2008) The global burden of disease: 2004 update. WHO library cataloguing-in-publication data.

12. Seck SM (2012) Issues of Renal Replacement Therapy in Elders Living Low-Income African Countries. Nephrourol Mon 4: 648-649.

13. Foley RN, Murray AM (2005) Chronic kidney disease and the risk for cardiovascular disease, renal replacement, and death in the United States Medicare population, 1998 to 1999. J Am Soc Nephrol 16: 489-495.

14. Kaze FF, Kengne AP, Mambap AT, Halle MP, Mbanya D, et al. (2015) Anemia in patients on chronic hemodialysis in Cameroon: Prevalence, characteristics and management in low resources setting. Afr Health Sci 15: 253-260.

15. Kaze FF, Halle MP, Mopa HT, Ashuntantang G, Fouda H, et al. (2015) Prevalence and risk factors of chronic kidney disease in urban adult Cameroonians according to three common estimators of the glomerular filtration rate: A cross-sectional study. BMC Nephrol.

16. Halle MP, Takongue C, Kengne AP, Kaze FF, Ngu KB (2015) Epidemiological profile of patients with end stage renal disease in a referral hospital in Cameroon. BMC Nephrol

17. Dzudie A, Kengne AP, Muna WFT, Ba H, Menanga A, et al. (2012) Prevalence, awareness, treatment and control of hypertension in a selfselected sub-Saharan African urban population: A crossectional study. BMJ.

18. Kamadjeu RM, Edwards R (2006) Anthropometry measures and prevalence of obesity in the urban adult population of Cameroon: An update from the Cameroon Burden of Diabetes Baseline Survey. BMC Public Health.

19. James MT, Hemmelgarn BR, Tonelli M (2010) Early recognition and prevention of chronic kidney disease. Lancet 375: 1296-1309. 